TRANSACTIONS OF THE

AMERICAN MATHEMATICAL SOCIETY

Volume 364, Number 3, March 2012, Pages 1281-1291

S 0002-9947(2011)05365-1

Article electronically published on November 7, 2011

\title{
A SUPPORT THEOREM FOR A GAUSSIAN RADON TRANSFORM IN INFINITE DIMENSIONS
}

\author{
JEREMY J. BECNEL AND AMBAR N. SENGUPTA
}

\begin{abstract}
We prove that in infinite dimensions, if a bounded, suitably continuous, function has zero Gaussian integral over all hyperplanes outside a closed bounded convex set, then the function is zero outside this set. This is an infinite-dimensional form of the well-known Helgason support theorem for Radon transforms in finite dimensions.
\end{abstract}

\section{INTRODUCTION}

The Radon transform [15] associates to a function $f$ on the finite-dimensional space $\mathbb{R}^{n}$ the function $R f$ on the set of all hyperplanes in $\mathbb{R}^{n}$ whose value on any hyperplane $P$ is the integral of $f$ over $P$ :

$$
R f(P)=\int_{P} f(x) d x
$$

the integration here being with respect to Lebesgue measure on $P$. This transform does not generalize directly to infinite dimensions because there is no useful notion of Lebesgue measure in infinite dimensions. However, there is a well-developed theory of Gaussian measures in infinite dimensions, and so it is natural to extend the Radon transform to infinite dimensions using Gaussian measure:

$$
G f(P)=\int f d \mu_{P}
$$

where $\mu_{P}$ is Gaussian measure for any hyperplane $P$ in a Hilbert space $H_{0}$. (By 'hyperplane' we shall always mean a translate of a closed linear subspace of codimension one.) This transform was developed in [14, but we shall present a self-contained account in section 2

A central feature of the classical Radon transform $R$ is the Helgason support theorem (Helgason [10]): if $f$ is a rapidly decreasing continuous function and $R f(P)$ is 0 on every hyperplane $P$ lying outside a compact convex set $K$, then $f$ is 0 off $K$. In this paper we prove an infinite-dimensional version of this support theorem.

A support theorem, even in the finite-dimensional case, works for a class of suitably regular functions, such as continuous functions of rapid decrease. In the infinite-dimensional setting it is first of all necessary to choose a framework for

Received by the editors November 4, 2009 and, in revised form, March 17, 2010 and April 6, 2010 .

2010 Mathematics Subject Classification. Primary 44A12; Secondary 28C20, 60H40.

The research of the first author was supported by National Security Agency Young Investigators Grant MPO-BA331.

The research of the second author was supported by US National Science Foundation Grant DMS-0601141.

(C)2011 American Mathematical Society Reverts to public domain 28 years from publication 
the Gaussian measures with respect to which the transform is defined (Bogachev [3] is an extensive account of the general theory of Gaussian measures on infinitedimensional spaces). There are two standard frameworks: (i) nuclear spaces and their duals [5, 6, 13]; (ii) Abstract Wiener Spaces [8, 12. For our purposes, we restrict to a more elementary setting, using only Hilbert spaces, as this highlights the essential ideas involved in proving our support theorem. Becnel [2] proves the result in the white noise analysis framework for a class of functions called Hida test functions.

The finite-dimensional Radon transform is of central significance in tomography (albeit here only the three-dimensional theory matters), and there is a vast body of results of purely mathematical interest, including one of the great early results which specifies the range of the space of Schwartz functions. The authors freely acknowldege that the theory in infinite dimensions is in its infancy and at this stage can only express their hope that larger developments, techniques, and possible applications lie in the future. The motivation for our investigation of the infinitedimensional theory arose in a stochastic context. Consider a random functional $F$, of suitable regularity, of a Brownian motion $t \mapsto B_{t}$. One may wish to recover information about $F$ from the conditional expectation values $\mathbb{E}\left[F \mid \int_{0}^{\infty} f(t) d B_{t}=\right.$ $c$, with $f$ running over a suitable collection of functions and $c$ over real numbers. Such a problem is essentially a problem concerning the Gauss-Radon transform in the setting of Gaussian measure over the Hilbert space $L^{2}([0, \infty))$. We shall not pursue this or other applications in the present paper where we develop the theory and central result in an abstract setting.

\section{The Gauss-Radon transform}

The Gauss-Radon transform $G f$ of a function $f$ for a real separable Hilbert space $H_{0}$ associates to each hyperplane $P$ in $H_{0}$ the integral of $f$ with respect to the Gaussian measure $\mu_{P}$ for the hyperplane $P$. Recall that by 'hyperplane' we mean a translate of a closed linear subspace of codimension one. In this section we will spell out the details of this, including a precise specification of the measure $\mu_{P}$ and the space on which $f$ is defined.

Throughout this paper $H_{0}$ is a separable real Hilbert space. The inner-product on $H_{0}$ will be denoted $\langle\cdot, \cdot\rangle_{0}$ or simply $\langle\cdot, \cdot\rangle$, and the corresponding norm by $\|\cdot\|_{0}$.

From well-known methods of Gaussian measure theory in infinite dimensions there is a measurable space $(\Omega, \mathcal{F})$ and a linear map $x \mapsto \hat{x}$ taking vectors $x$ in some dense subspace $H_{1}$ of $H_{0}$ to measurable functions on $\Omega$, such that for any closed subspace $F \subset H_{0}$ and any $u \in F$, there is a probability measure $\mu_{u+F^{\perp}}$ on $(\Omega, \mathcal{F})$ satisfying

$$
\int e^{i \hat{x}} d \mu_{u+F^{\perp}}=e^{i\langle x, u\rangle_{0}-\frac{1}{2}\left\|x_{F} \perp\right\|_{0}^{2}}
$$

where $x_{F^{\perp}}$ denotes the orthogonal projection of $x$ onto $F^{\perp}$. See, for instance, Kuo [12, 13, and Gelfand et al. [6] for Gaussian measures in infinite dimensions and Bogachev [3, Theorem 3.10.2] for Gaussian measures in infinite-dimensionals spaces and on hyperplanes of such spaces.

There are several possible choices for the space $(\Omega, \mathcal{F})$. In this paper we will stay within the Hilbert-space setting (which goes back, in a more general context, to Sazonov [17] and Gross [9]) and make the following choice. Fix, once and for all, 
a Hilbert-Schmidt operator $T: H_{0} \rightarrow H_{0}$ with $0 \leq T \leq I$, and let

$$
H_{1}=T^{1 / 2}\left(H_{0}\right)
$$

with Hilbert space structure given by the inner-product

$$
\langle x, y\rangle_{1}=\left\langle T^{-1 / 2} x, T^{-1 / 2} y\right\rangle_{0} .
$$

Identifying $H_{0}$ with its dual $H_{0}^{\prime}$ in the usual way produces Hilbert-Schmidt inclusions

$$
H_{1} \subset H_{0} \simeq H_{0}^{\prime} \subset H_{-1} \stackrel{\text { def }}{=} H_{1}^{\prime},
$$

where $H_{-1}$ is the dual space to the Hilbert space $H_{1}$. Note that $\|\cdot\|_{0} \leq\|\cdot\|_{1}$ on $H_{1}$ and $\|\cdot\|_{-1} \leq\|\cdot\|_{0}$ on $H_{0}$. The measurable space $(\Omega, \mathcal{F})$ is then just $H_{-1}$ with its Borel $\sigma$-algebra. For $x \in H_{1}$ we take $\hat{x}$ on $H_{-1}$ to be the evaluation map $x^{\prime} \mapsto\left\langle x^{\prime}, x\right\rangle$.

The characteristic function of $\mu_{u+F^{\perp}}$ provided by (2.1) implies that, with respect to the probability measure $\mu_{u+F^{\perp}}$, the random variable $\hat{x}$ has Gaussian distribution with mean $\langle x, u\rangle_{0}$ and variance $\left\|x_{F^{\perp}}\right\|_{0}^{2}$. Hence,

$$
\|\hat{x}\|_{L^{2}\left(\mu_{u+F^{\perp}}\right)}^{2}=\left|\langle u, x\rangle_{0}\right|^{2}+\left\|x_{F^{\perp}}\right\|_{0}^{2} \leq\left(\|u\|_{0}^{2}+1\right)\|x\|_{0}^{2} .
$$

Thus, $x \mapsto \hat{x}$ is continuous as a map $H_{-1} \rightarrow L^{2}\left(H_{-1}, \mu_{u+F^{\perp}}\right)$ and so extends to a continuous linear map

$$
H_{0} \rightarrow L^{2}\left(\mu_{u+F^{\perp}}\right): x \mapsto \hat{x},
$$

with $\hat{x}$ satisfying (2.1), i.e. $\hat{x}$ is a Gaussian variable with mean $\langle x, u\rangle_{0}$ and variance $\left\|x_{F^{\perp}}\right\|_{0}^{2}$, and hence also the bound (2.5). Note, however, that for $x$ in $H_{0}$ outside $H_{-1}$, the definition of $\hat{x}$ depends on $\mu_{u+F^{\perp}}$ and hence on the affine subspace $u+F^{\perp}$.

The measure $\mu_{u+F^{\perp}}$ is indeed concentrated 'on' the affine subspace $u+F^{\perp}$, in the sense that $\hat{x}$, for $x \in F$, is equal to the constant $\langle x, u\rangle_{0}$ almost surely with respect to the measure $\mu_{u+F^{\perp}}$.

Definition 2.1. If $f$ is a bounded Borel measurable function on $H_{-1}$, then its Gauss-Radon transform $G f$ is the function which associates to each hyperplane $P$ in $H_{0}$ the value

$$
G f(P)=\int_{H_{-1}} f d \mu_{P} .
$$

To stress the role of $H_{0}$, we may sometimes write $G_{H_{0}}$ instead of $G$.

\section{Some GeOmetric And Limiting Results}

We work within the framework of infinite-dimensional Hilbert spaces $H_{1} \subset H_{0} \subset$ $H_{-1}$ described before and denote the closed ball of radius $R$ in $H_{-1}$ by $D_{-1}(R)$ :

$$
D_{-1}(R)=\left\{x^{\prime} \in H_{-1}:\left\|x^{\prime}\right\|_{-1} \leq R\right\} .
$$

Proposition 3.1. Let $F$ be a finite-dimensional subspace of the separable real Hilbert space $H_{0}$, and let $U$ be a bounded subset of $F$. Then for any $\epsilon>0$, there is an $R \in(0, \infty)$ such that

$$
\mu_{u+F^{\perp}}\left[D_{-1}(R)\right]>1-\epsilon,
$$

for all $u \in U$. 
Proof. Fix an orthonormal basis $v_{1}, v_{2}, \ldots$ of the Hilbert space $H_{1}$. The complement $D_{-1}(R)^{c}$ is given by

$$
D_{-1}(R)^{c}=\left[\frac{1}{R^{2}} \sum_{n=1}^{\infty} \hat{v}_{n}^{2}>1\right] \subset H_{-1} .
$$

So, as in Chebyshev's inequality,

$$
\begin{aligned}
\mu_{u+F^{\perp}}\left[D_{-1}(R)^{c}\right] & \leq \int_{H_{-1}} \frac{1}{R^{2}} \sum_{n=1}^{\infty} \hat{v}_{n}^{2} d \mu_{u+F^{\perp}} \\
& =\frac{1}{R^{2}} \sum_{n=1}^{\infty}\left\|\hat{v}_{n}\right\|_{L^{2}\left(\mu_{u+F^{\perp}}\right)}^{2} \\
& \left.\leq \frac{1}{R^{2}} \sum_{n=1}^{\infty}\left(\|u\|_{0}^{2}+1\right)\left\|v_{n}\right\|_{0}^{2} \quad \text { (by (2.5) }\right) \\
& \leq \frac{1}{R^{2}}\left(\sum_{n=1}^{\infty}\left\|v_{n}\right\|_{-1}^{2}\right)\left(\sup _{u \in U}\|u\|_{0}^{2}+1\right),
\end{aligned}
$$

which is $<\epsilon$ when $R$ is large enough, since $U$ is bounded and $\sum_{n=1}^{\infty}\left\|v_{n}\right\|_{-1}^{2}<\infty$.

Here is a convenient way to look at integrals over affine subspaces:

Proposition 3.2. If $F$ is a closed subspace of the real separable Hilbert space $H_{0}$ and $u \in F$, then

$$
\int f\left(x^{\prime}\right) d \mu_{u+F^{\perp}}\left(x^{\prime}\right)=\int f\left(x^{\prime}+u\right) d \mu_{F^{\perp}}\left(x^{\prime}\right)
$$

whenever $f$ is a measurable function on $H_{-1}$, and the equality here holds in the sense that if either side is defined, so is the other, and the integrals are then equal.

This result is checked for $f=e^{i \hat{x}}$, with $x \in H_{-1}$, and then extends to general $f$ by routine arguments. Observe that $F$ sits inside $H_{0}$, which we are viewing as a subspace of $H_{1}^{\prime}$. Thus $u \in F$ is also a function on $H_{1}$, mapping any $x \in H_{1}$ to $\langle u, x\rangle_{0}$.

Proposition 3.3. Suppose that $f: H_{-1} \rightarrow \mathbb{R}$ is a bounded function on the Hilbert space $H_{-1}$ which is either uniformly continuous in the Hilbert $\|\cdot\|_{-1}$-norm topology or sequentially continuous in the weak dual topology on $H_{1}^{\prime}$. Then for every finitedimensional subspace $F$ of $H_{0}$, the function $u \mapsto \int f d \mu_{u+F^{\perp}}$ is continuous and bounded in $u \in F$.

The version with weak sequential continuity will not be needed for our main result, Theorem 4.1, but is useful for the analogous result for nuclear spaces.

Proof. Let $U$ be a bounded neighborhood of $u$ in $F$. Let $\epsilon>0$. Then, by Proposition 3.1, there is an $R \in(0, \infty)$ such that

$$
\mu_{y+F^{\perp}}\left[D_{-1}(R)\right]>1-\epsilon
$$


for all $y \in U$, where $D_{-1}(R)$ is the closed $R$-ball in $H_{-1}$. Then, for any $v \in U$, we have

$$
\begin{aligned}
\left|\int f d \mu_{u+F^{\perp}}-\int f d \mu_{v+F^{\perp}}\right| & \leq \int_{D_{-1}(R)}\left|f\left(x^{\prime}+u\right)-f\left(x^{\prime}+v\right)\right| d \mu_{F^{\perp}}\left(x^{\prime}\right) \\
& \leq \sup _{x^{\prime} \in D_{-1}(R)}\left|f\left(x^{\prime}+u\right)-f\left(x^{\prime}+v\right)\right|+2 \epsilon\|f\|_{\text {sup }}
\end{aligned}
$$

Now we claim uniform continuity: there is a $\delta>0$ such that if $v \in F$ with $\|v-u\|_{0}<$ $\delta$, then $\sup _{x^{\prime} \in D_{-1}(R)}\left|f\left(x^{\prime}+u\right)-f\left(x^{\prime}+v\right)\right| i s<\epsilon$. Of course, this is given if $f$ is uniformly continuous in the strong topology (note that $v-u \in F$, and, of course, there the norms $\|\cdot\|_{1}$ and $\|\cdot\|_{0}$ are equivalent on $F$ ). Now suppose $f$ is sequentially continuous in the weak topology, and assume that the uniform continuity claim does not hold. Then there is a sequence of points $v_{n} \in F$ converging to $u$ and a sequence of points $x_{n}^{\prime} \in D_{-1}(R)$ such that $\left|f\left(x_{n}^{\prime}+u\right)-f\left(x_{n}^{\prime}+v_{n}\right)\right|$ is $\geq \epsilon$. Now, by the Banach-Alaoglu theorem and separability, $D_{-1}(R)$ is compact and metrizable in the weak topology of $H_{-1}=H_{1}^{\prime}$, and so we may assume that $x_{n}^{\prime} \rightarrow x^{\prime}$ weakly for some $x^{\prime} \in D_{-1}(R)$. Hence also $x_{n}^{\prime}+v_{n} \rightarrow x^{\prime}+u$, and so

$$
f\left(x_{n}^{\prime}+u\right)-f\left(x_{n}^{\prime}+v_{n}\right) \rightarrow f(x+u)-f(x+u)=0,
$$

contradicting the assumption made.

Thus, indeed, for all $v$ in some neighborhood of $u$ in $F$ we have

$$
\sup _{x^{\prime} \in D_{-1}(R)}\left|f\left(x^{\prime}+u\right)-f\left(x^{\prime}+v\right)\right|<\epsilon .
$$

Then we have for such $v$, from (3.6),

$$
\left|\int f d \mu_{u+F^{\perp}}-\int f d \mu_{v+F^{\perp}}\right| \leq\left(1+2\|f\|_{\text {sup }}\right) \epsilon,
$$

which shows that $\int f d \mu_{u+F^{\perp}}$ depends continuously on $u \in F$.

Next we have a key limiting result:

Proposition 3.4. Suppose that $f$ is a bounded Borel function on $H_{-1}$, continuous at $u \in H_{0} \subset H_{-1}$ relative to the norm $\|\cdot\|_{-1}$. Then

$$
\lim _{u \in F \rightarrow H_{0}} \int f d \mu_{u+F^{\perp}}=f(u),
$$

in the sense that if $F_{1} \subset F_{2} \subset \cdots$ is a sequence of finite-dimensional subspaces, with $u \in F_{1}$, such that $\bigcup_{n \geq 1} F_{n}$ is dense in $H_{0}$, then

$$
\lim _{n \rightarrow \infty} \int f d \mu_{u+F_{n}^{\perp}}=f(u) .
$$

Proof. Let $\epsilon>0$. Then there is a closed ball $D_{-1}(R)$ in the Hilbert space $H_{-1}$, centered at 0 and having radius some $R \in(0, \infty)$, such that

$$
\sup _{x^{\prime} \in D_{-1}(R)}\left|f\left(u+x^{\prime}\right)-f(u)\right|<\epsilon .
$$


Let $v_{1}, v_{2}, \ldots$ be an orthonormal basis of the Hilbert space $H_{1}$. For any finitedimensional subspace $F$ in $H_{0}$, we have

$$
\begin{aligned}
\mu_{F^{\perp}}\left(D_{-1}(R)^{c}\right)=\mu_{F^{\perp}}\left[\sum_{m=1}^{\infty} \hat{v}_{m}^{2}>R^{2}\right] & \leq \frac{1}{R^{2}} \sum_{m=1}^{\infty} \int \hat{v}_{m}^{2} d \mu_{F^{\perp}} \\
& \left.=\frac{1}{R^{2}} \sum_{m=1}^{\infty}\left\|\left(v_{m}\right)_{F^{\perp}}\right\|_{0}^{2} \quad \text { (by (2.5) }\right) .
\end{aligned}
$$

Note that this series is dominated termwise by the convergent series $\sum_{m=1}^{\infty}\left\|v_{m}\right\|_{0}^{2}$.

Now, to apply this to the subspaces $F_{n}$, observe first that the projection $x_{F_{n}^{\perp}}=$ $x-x_{F_{n}}$ converges to 0 . Hence, by dominated convergence,

$$
\lim _{n \rightarrow \infty} \sum_{m=1}^{\infty}\left\|\left(v_{m}\right)_{F_{n}^{\perp}}\right\|_{0}^{2}=0
$$

Consequently,

$$
\lim _{n \rightarrow \infty} \mu_{F_{n}^{\perp}}\left(D_{-1}(R)^{c}\right)=0 .
$$

Now

$$
\begin{aligned}
\left|\int f d \mu_{u+F_{n}^{\perp}}-f(u)\right| & \leq \int\left|f\left(u+x^{\prime}\right)-f(u)\right| d \mu_{F_{n}^{\perp}}\left(x^{\prime}\right) \\
& \leq \sup _{x^{\prime} \in D_{-1}(R)}\left|f\left(u+x^{\prime}\right)-f(u)\right|+2\|f\|_{\sup } \mu_{F_{n}^{\perp}}\left(D_{-1}(R)^{c}\right) .
\end{aligned}
$$

By the choice of $R$, the first term on the right is $<\epsilon$, as noted in (3.9). Next, with this $R$, the second term is $<\epsilon$ when $n$ is large enough. Hence we have the limiting result (3.8).

Subspaces $X$ and $Y$, and any of their translates, of a Hilbert space are said to be perpendicular if neither is a subspace of the other and they can be split into mutually orthogonal subspaces

$$
X=(X \cap Y)+\left(X \cap Y^{\perp}\right) \quad \text { and } \quad Y=(X \cap Y)+\left(X^{\perp} \cap Y\right) .
$$

This means that a vector in $X$ (or $Y$ ) which is orthogonal to $X \cap Y$ is in fact orthogonal to $Y$ (or $X)$.

Proposition 3.5. Consider a hyperplane $P$ in the infinite-dimensional Hilbert space $H_{0}$ and a finite-dimensional subspace $F \neq\{0\}$ of $H_{0}$. Then $P$ and $F$ are perpendicular if and only if $P$ can be expressed as

$$
P=u+u_{0}^{\perp}
$$

for some non-zero vector $u_{0} \in F$ and $u$ is a multiple of $u_{0}$. Moreover, $P \cap F$ is a hyperplane within the finite-dimensional space $F$.

We omit the proof, which is elementary.

The following relates the Gauss-Radon transform in infinite dimensions to that for finite-dimensional subspaces by a disintegration process. 


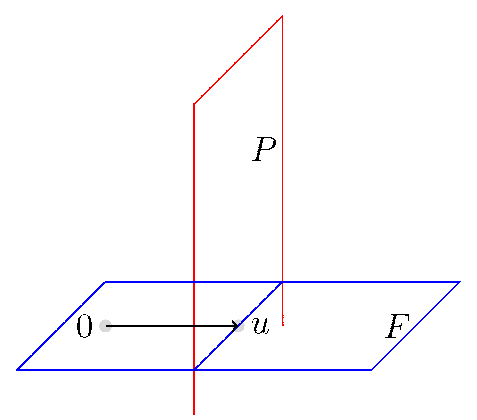

Figure 1. A hyperplane $P$ intersecting a subspace $F$

Proposition 3.6. Let $P$ be a hyperplane in $H_{0}$, and let $F \neq 0$ be a finitedimensional subspace of $H_{0}$ which is perpendicular to $P$. Then for any bounded Borel function $f$ on $H_{-1}$ we have

$$
G f(P)=G_{F}\left(f^{*}\right)(P \cap F),
$$

where, on the right, $G_{F}\left(f^{*}\right)$ is the Gauss-Radon transform, within the subspace $F$, of the function $f^{*}$ on $F$ given by

$$
f^{*}(y)=\int_{H_{-1}} f d \mu_{y+F^{\perp}}
$$

Part of the conclusion here is that $f^{*}$ is a measurable function on $F$.

Proof. Let $u_{0}$ be a non-zero vector in $F$ orthogonal to the hyperplane $P$, and let $u$ be the point on $P \cap F$ closest to the origin. Then (see Figure 1 with $u_{0}$ along $u$ ):

$$
P \cap F=u+\left(u_{0}^{\perp} \cap F\right) .
$$

It will suffice to prove the result for a function of the form $\phi=e^{i \hat{x}}$, where $x \in H_{1}$. For such $\phi$, we have

$$
\begin{aligned}
G_{F}\left(\phi^{*}\right)(P \cap F) & =\int_{F}\left(\int_{\mathcal{H}^{\prime}} e^{i \hat{x}} d \mu_{y+F^{\perp}}\right) d \mu_{P \cap F}(y) \\
& =\int_{F} e^{i\langle x, y\rangle_{0}-\frac{1}{2}\left\|x_{F}\right\|_{0}^{2}} d \mu_{u+\left(u_{0}^{\perp} \cap F\right)}(y) \\
& =e^{-\frac{1}{2}\left\|x_{F} \perp\right\|_{0}^{2}} \int e^{i\left\langle x_{F}, y\right\rangle_{0}} d \mu_{u+\left(u_{0}^{\perp} \cap F\right)}(y) \\
& =e^{-\frac{1}{2}\left\|x_{F} \perp\right\|_{0}^{2}+i\left\langle x_{F}, u\right\rangle_{0}-\frac{1}{2}\left\|x_{u_{0}^{\perp} \cap F}\right\|_{0}^{2}} \\
& =e^{i\langle x, u\rangle_{0}-\frac{1}{2}\left\|x_{u_{0}^{\perp}}\right\|^{2}},
\end{aligned}
$$

where, in the last step, we used the fact that the component of $x_{u_{0}^{\perp}}$ orthogonal to $u_{0}^{\perp} \cap F$ is simply $x_{F^{\perp}}$. This is because $u_{0}^{\perp}$ and $F$ are perpendicular so that a vector in $u_{0}^{\perp}$ which is orthogonal to $u_{0}^{\perp} \cap F$ is just a vector in $u_{0}^{\perp}$ orthogonal to $F$. Comparing with $G \phi(P)=e^{i\langle x, u\rangle_{0}-\frac{1}{2}\left\|x_{u_{0}^{\perp}}\right\|_{0}^{2}}$, we see that the result is proved for $\phi=e^{i \hat{x}}$. The general case now follows by routine arguments. 
Lastly we have a geometric observation:

Proposition 3.7. Let $K_{0}$ be a closed convex subset of the Hilbert space $H_{0}$, and let $v$ be a point outside $K_{0}$. Then there is a finite-dimensional subspace $F_{0} \subset H_{0}$ containing $v$, and a sequence of finite-dimensional subspaces $F_{n} \subset H_{0}$ with $F_{0} \subset$ $F_{1} \subset \cdots$ and $\bigcup_{n \geq 0} F_{n}$ dense in $H_{0}$ such that $v+F_{n}^{\perp}$ is disjoint from $K_{0}$ for each $n \in\{1,2,3, \ldots\}$. Moreover, $v$ lies outside the orthogonal projection $\operatorname{pr}_{F_{n}}\left(K_{0}\right)$ of $K_{0}$ onto $F_{n}$ :

$$
v \notin \operatorname{pr}_{F_{n}}\left(K_{0}\right) \quad \text { for all } n \in\{1,2,3, \ldots\} .
$$

Proof. Since $K_{0}$ is closed and convex in the Hilbert space $H_{0}$, there is a unique point $v_{0} \in K_{0}$ closest to $v$. Then the hyperplane through $v$ orthogonal to the vector $u_{0}=v-v_{0}$, i.e. the hyperplane $v+u_{0}^{\perp}$, does not contain any point of $K_{0}$. For, otherwise, there would be some $w \in u_{0}^{\perp}$ with $v+w$ in $K_{0}$, and then in the right angled triangle

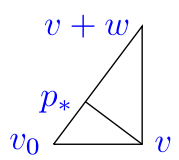

formed by the points $v_{0}, v$, and $v+w$ (which has a right angle at the point $v$ ) there would be a point $p_{*}$ on the hypotenuse, joining $v_{0}$ and $v+w$, and hence lying in the convex set $K$, which would be closer to $v$ than is $v_{0}$.

Let $F_{0}$ be the subspace of $H_{0}$ spanned by the vectors $v_{0}$ and $u_{0}$; note that $v \in F_{0}$. Now choose an orthonormal basis $u_{1}, u_{2}, \ldots$ of the closed subspace $F_{0}^{\perp}$, and let

$$
F_{n}=F_{0}+\text { linear span of } u_{1}, \ldots, u_{n} .
$$

This gives an increasing sequence of finite-dimensional subspaces whose union contains $F_{0}$ as well as all the vectors $u_{n}$, and hence is dense in $H_{0}$. Next observe that

$$
v+F_{n}^{\perp} \subset v+F_{0}^{\perp} \subset v+u_{0}^{\perp} .
$$

As noted before, $v+u_{0}^{\perp}$ is disjoint from $K_{0}$, and so $v+F_{n}^{\perp}$ is disjoint from $K_{0}$.

Since the hyperplane $v+u_{0}^{\perp}$ is precisely the set of points in $H_{0}$ whose innerproduct with $u_{0}$ equals $\left\langle u_{0}, v\right\rangle$, it follows that no point in $K_{0}$ has an inner-product with $u_{0}$ equal to $\left\langle u_{0}, v\right\rangle$. In particular, the orthogonal projection of $K_{0}$ on $F_{n}$ cannot contain $v$, for if a point $p$ in $K_{0}$ projected orthogonally onto $F_{n}$ produced $v$, then its inner-product $\left\langle u_{0}, p\right\rangle$ with $u_{0}$ would be the same as $\left\langle u_{0}, v\right\rangle$. This proves (3.16).

\section{THE SUPPORT THEOREM}

We continue with the framework set up in the preceding sections, and now turn to our main result. Thus, $H_{0}$ is a separable, infinite-dimensional real Hilbert space, which is a Hilbert-Schmidt completion of a Hilbert space $H_{1} \subset H_{0}$, and $H_{-1}$ is the dual $H_{1}^{\prime}$.

Theorem 4.1. Suppose that $f: H_{-1} \rightarrow \mathbb{R}$ is a bounded function on the Hilbert space $H_{-1}$ which is uniformly continuous in the strong (Hilbert) topology on $H_{-1}$. Let $K_{0}$ be a closed, bounded, convex subset of $H_{0}$. If the Gauss-Radon transform 


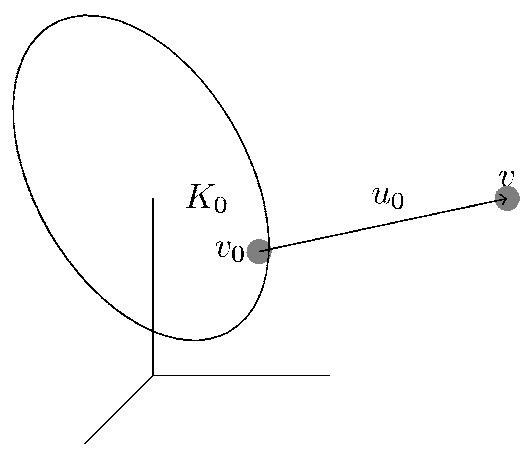

Figure 2. A compact convex set $K_{0}$ and a point $v$ outside it

of $f$ is 0 on hyperplanes which do not intersect $K_{0}$, then $f$ is 0 on the complement of $K_{0}$ in $H_{0}$.

Proof. We work with a point $v \in H_{0}$ outside $K_{0}$.

In Proposition 3.7 we constructed a sequence of finite-dimensional subspaces $F_{n} \subset H_{0}$ containing $v$, with $F_{1} \subset F_{2} \subset \cdots$ such that $\bigcup_{n \geq 1} F_{n}$ is dense in $H_{0}$ and $v+F_{n}^{\perp}$ is disjoint from $K_{0}$ for every positive integer $n$. Recall briefly how this was done. First we chose a point $u \in K_{0}$ closest to $v$, we set

$$
u_{0}=v-u,
$$

then we chose $u_{1}, u_{2}, \ldots$ an orthonormal basis of $u_{0}^{\perp}$, set $F_{0}$ to be the linear span of $v$ and $u_{0}$, and took $F_{n}$ to be the linear span of $v, u_{0}, u_{1}, \ldots, u_{n}$. We showed that the hyperplane

$$
v+u_{0}^{\perp}
$$

is disjoint from $K_{0}$ and that $v$ is outside the orthogonal projection $\operatorname{pr}_{F_{n}}\left(K_{0}\right)$ of $K_{0}$ onto $F_{n}$.

Let $f_{F_{n}}^{*}$ be the function on $F$ given by

$$
f_{F_{n}}^{*}(x)=\int f d \mu_{x+F_{n}^{\perp}} .
$$

We will show that this is 0 whenever $x$ lies outside $\operatorname{pr}_{F_{n}}\left(K_{0}\right)$. In particular, it will follow that $f_{F_{n}}^{*}(v)$ is 0 for all $n \in\{1,2,3, \ldots\}$, and so, by the limiting result of Proposition 3.4

$$
f(v)=\lim _{n \rightarrow \infty} f_{F_{n}}^{*}(v)=0 .
$$

We drop the subscript $n$ in the following, and work with a finite-dimensional space $F \subset H_{0}$ for which

$$
v \in F \text {, but } v \notin \operatorname{pr}_{F}\left(K_{0}\right) \text {. }
$$

Let $P^{\prime}$ be a hyperplane within the subspace $F$. Then

$$
P^{\prime}=P \cap F,
$$

where $P$ is the hyperplane in $H_{0}$ given by

$$
P=P^{\prime}+F^{\perp},
$$




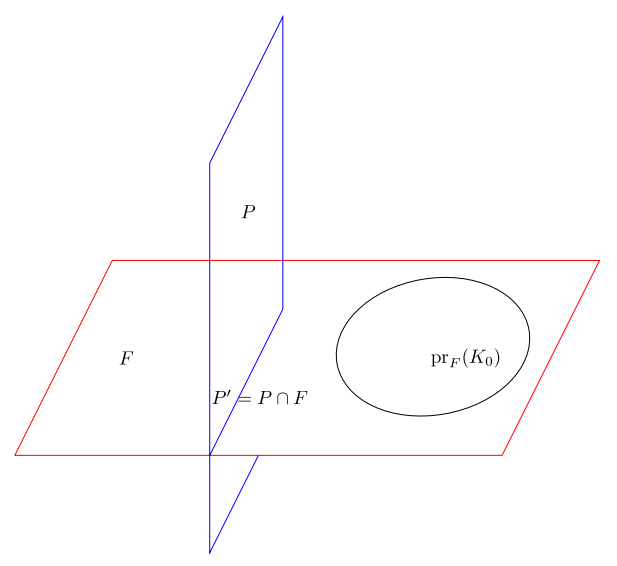

Figure 3 . The projection of a compact set $K_{0}$ on $F$, disjoint from a hyperplane $P^{\prime}=P \cap F$ in $P$

which is perpendicular to $F$. If $P^{\prime}$ is disjoint from $\operatorname{pr}_{F}\left(K_{0}\right)$, then $P$ is disjoint from $K_{0}$ because any point in $P \cap K_{0}$ projects by $\operatorname{pr}_{F}$ to a point which is both $P^{\prime}$ and $\operatorname{pr}_{F}\left(K_{0}\right)$. Now recall the disintegration formula (3.14) says

$$
G f(P)=G_{F}\left(f_{F}^{*}\right)\left(P^{\prime}\right) .
$$

By hypothesis, this is 0 if $P$ is disjoint from $K_{0}$, i.e. if $P^{\prime}$ is disjoint from $\operatorname{pr}_{F}\left(K_{0}\right)$. Now observe that $\operatorname{pr}_{F}\left(K_{0}\right)$ is a convex, compact subset of $F$ (compactness follows because $K_{0}$, being convex, closed and bounded is weakly compact, and hence any finite-dimensional projection is compact). Note that by Proposition 3.3 , the function $f_{F}$ is continuous on $F$ and is, of course, also bounded. It then follows by the finite-dimensional Radon transform support theorem (applied to $f_{F}$ times a Gaussian density) that $f_{F}^{*}$ is 0 outside $\operatorname{pr}_{F}\left(K_{0}\right)$. In particular, $f_{F}^{*}(v)$ is 0 , which is what was needed to complete the proof.

4.1. Support theorem in nuclear spaces. As noted in the introduction, there are two frameworks for analysis in infinite dimensions used widely: (i) Abstract Wiener Spaces [8, 12, and (ii) Nuclear spaces. The latter form a useful setting for both quantum field theory (for instance [7, 16]) and stochastic analysis (for instance [4, 11]). Our results have been framed entirely in terms of Hilbert spaces, but they extend readily to the nuclear space setting. Suppose $H_{0}$ is a real separable Hilbert space, $T: H_{0} \rightarrow H_{0}$ is a Hilbert-Schmidt operator, with $0 \leq T \leq I, H_{p}=T^{p / 2}\left(H_{0}\right)$ for $p \in\{0,1,2, .$.$\} and \mathcal{H}=\bigcap_{p \in\{0,1,2, . .\}} H_{p}$, equipped with the topology generated by the norms given by $\|x\|_{p}=\left\|T^{-p / 2} x\right\|_{0}$. The dual $\mathcal{H}^{\prime}$ of the 'nuclear' space $\mathcal{H}$ has the weak topology as well as a strong topology [5, 6, and the hyperplane measures $\mu_{u+F^{\perp}}$ may be realized on $\mathcal{H}^{\prime} \supset H_{-1}=H_{1}^{\prime}$. In the space $\mathcal{H}^{\prime}$, a strongly continuous function is strongly sequentially continuous. This is equivalent to weak sequential continuity, and so Proposition 3.3 is applicable. Then the support theorem says that if $f$ is a bounded, strongly continuous function on $\mathcal{H}^{\prime}$ whose Gauss-Radon transform vanishes on hyperplanes lying outside a convex, closed and bounded set $K_{0} \subset H_{0}$, then $f$ is 0 off $K_{0}$. 


\section{ACKNOWLEDGMENTS}

Our thanks to Hui-Hsiung Kuo for discussions on infinite-dimensional spaces, and to Gestur Ólafsson for a history of the finite-dimensional support theorem. At the suggestion of the anonymous referee, we recast our original arguments from the nuclear space setting to the Hilbert space framework.

\section{REFERENCES}

1. Becnel, Jeremy J.: Equivalence of Topologies and Borel Fields for Countably-Hilbert Spaces, Proceedings of the American Mathematical Society, 134 (2006), pp. 581-590 MR2176027 (2006j:57044)

2. Becnel, Jeremy J.: A Support Theorem for the Gauss-Radon Transform, 2009.

3. Bogachev, Vladimir I.: Gaussian Measures (Mathematical Surveys and Monographs), American Mathematical Society (1998). MR.1642391 (2000a:60004)

4. Chiang, T. S., Kallianpur, Gopinath, and Sundar, P.: Propagation of Chaos and the McKeanVlasov Equation in Duals of Nuclear Spaces, Appl Math Optim 24:55-83 (1991) MR.1106926 (92g:60078)

5. Gel'fand, I.M., and Shilov, G. E: Generalized Functions (Transl. M. D. Friedman, A. Feinstein, C. P. Peltzer) Academic Press, Volume 2 (1968) MR0230128 (37:5693)

6. Gel'fand, I.M., and Vilenkin, N. Ya.: Generalized Functions (Transl. A. Feinstein) Academic Press, Volume 4 (1964) MR0173945 (30:4152)

7. Glimm, James, and Jaffe, Arthur: Quantum physics: a functional integral point of view, 2nd Ed, New York : Springer-Verlag, 1987. MR887102 (89k:81001)

8. Gross, Leonard: Abstract Wiener spaces, Proc. 5th Berkeley Symp. Math. Stat. Probab., 2 (1965) pp. 31-42. MR0212152 (35:3027)

9. Gross, Leonard: Harmonic analysis on Hilbert space, Mem. Amer. Math. Soc. No. 461963. MR 0161095 (28:4304)

10. Helgason, Sigurdur: The Radon Transform. Birkhäuser (1980). MR573446 (83f:43012)

11. Itô, Kiyosi: Foundations of Stochastic Differential Equations in Infinite Dimensional Spaces, CBMS-NSF Regional Conference Series in Applied Mathematics 47, Society for Industrial and Applied Mathematics, 1984. MR771478 (87a:60068)

12. Kuo, Hui-Hsiung: Gaussian measures in Banach spaces, Springer Lecture Notes in Mathematics Vol 463, Spring-Verlag (1975). MR0461643 (57:1628)

13. Kuo, Hui-Hsiung: White noise distribution theory, CRC Press (1996). MR 1387829 (97m:60056)

14. Mihai, Vochita, and Sengupta, Ambar N.: The Radon-Gauss transform, Soochow J. Math. 33, 415-434 (2007). Online at http://www.math.lsu.edu/ ${ }^{\text {sengupta/papers/ }}$ mihaisengJune2007.pdf. MR2344371 (2009a:44006)

15. Radon, J.: Über die Bestimmung von Funktionen durch ihre Integralwerte längs gewisser Mannigfaltigkeiten. Ber. Verh. Sächs. Akad. Wiss. Leipzig, Math-Nat. kl. 69 (1917), 262-277.

16. Rivasseau, Vincent: From peturbative to constructive renormalization, Princeton University Press, 1991. MR 1174294 (93i:81004)

17. Sazonov, V: On characteristic functionals. (Russian) Teor. Veroyatnost. i Primenen. 31958 201-205. MR0098423 (20:4882)

Department of Mathematics and Statistics, Stephen F. Austin State University, Nacogdoches, Texas 75962-3040

E-mail address: becneljj@sfasu.edu

Department of Mathematics, Louisiana State University, Baton Rouge, Louisiana 70803

E-mail address: sengupta@gmail.com 